Review (Narrative)

\title{
A Review of the Circle of Willis \\ Investigative Methods, Anatomical Variations and Correlated Ischemic Brain Diseases
}

Wei Wang, Qiudian Wang, Guanying Yang, Mingrui Qiu, Aizhen Pan

\section{SUMMARY}

The Circle of Willis (CoW) is the most important collateral pathway communicating between the bilateral carotid system and the posterior circulation. Many techniques have been used to investigate the configuration and function of the CoW and their advantages and disadvantages are reviewed here. In previous studies, morphometric variation in CoW has been widely detected; however, the frequency of variation ranges largely, between $10 \%$ and $85 \%$. Some differences in reported frequency may reflect differences in: the definition of CoW variation, the methods used to examine variation and study populations. Two conclusions, however, are clear: (i) the prevalence of variation in the posterior circle is higher than in that of the anterior circle; and (ii) variations in the CoW are correlated with cerebral or carotid vessel diseases. Although the cause of the variants remains unclear, both genetic background and hemodynamics could play important roles. Lastly, the correlation between the CoW and ischemic brain diseases is discussed. In future, artificial intelligence will be helpful for evaluating the CoW.

\section{KEYWORDS}

Circle of Willis; Diagnostic Imaging; Dissection; Anatomic Variation;

Brain Ischemia

Sci Insigt. 2020; 33(2):159-166. doi:10.15354/si.20.re069.

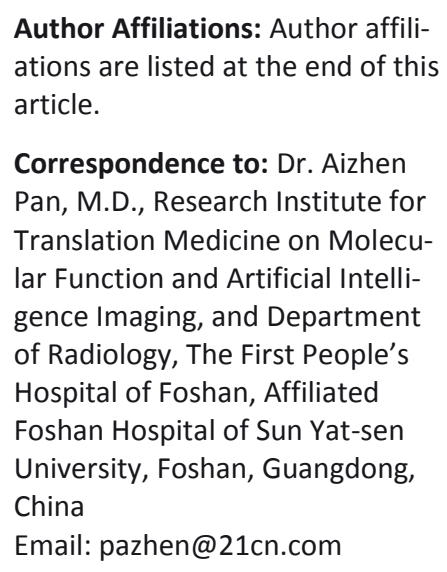

Author Affiliations: Author affiliations are listed at the end of this article.
Correspondence to: Dr. Aizhen Pan, M.D., Research Institute for Translation Medicine on Molecu- lar Function and Artificial Intelli- gence Imaging, and Department of Radiology, The First People's Hospital of Foshan, Affiliated Foshan Hospital of Sun Yat-sen University, Foshan, Guangdong, Email: pazhen@21cn.com




\section{DEFINITION AND IMPORTANCE OF THE CIR- CLE OF WILLIS}

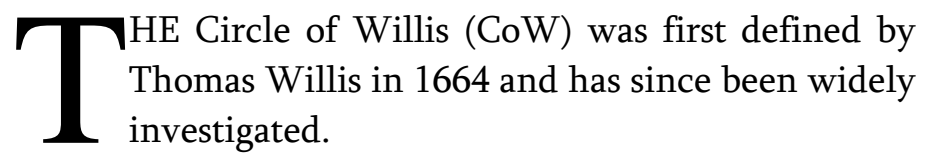

Most of artery distribution in the brain originates from CoW, which is the most important collateral pathway. The knowledge for $\mathrm{CoW}$ is the basis for the research of cerebral hemodynamic and is significant helpful for the prevention and treatment of stroke and cerebral vessel-related diseases. However, there are still some questions. There are more than 20 types of variation in the CoW and the reported frequency of variation in CoW are significant different among previous literature, from $10 \%$ to $85 \%$. Why the variation is so common and what the reason is? The relationship study between CoW and cerebral disease is rare. In the review, we focus on the variation in the CoW and demonstrate the latest research results. We hope to clarify the significance of variation in CoW and its role in ischemic cerebral disease.

During embryonic development, the complete form of the CoW appears on day 52. The CoW is located at the base of the brain, projecting from the terminus of the bilateral internal carotid arteries and the basilar artery (1). The typical configuration of the $\mathrm{CoW}$ is a ring formed by several vascular segments, including bilateral precommunicating part of bilateral anterior cerebral artery (A1), bilateral precommunicating part of bilateral posterior cerebral artery (P1), slender bilateral posterior communicating arteries (Pco) and an anterior communicating artery (Aco). The bilateral A1s and Aco form the anterior part of the CoW and the bilateral P1s and Pco form the posterior part of CoW. However, a high rate of variation in the $\mathrm{CoW}$ is found across populations (2), with either absence or hypoplasticity of segments observed. Functionally, along with the ophthalmic artery and leptomeningeal vessels, the CoW supports the redistribution of blood supply in brain; the CoW is thought to play the most important role. In the case of a decreased blood supply from afferent arteries, an intact CoW can direct blood flow from other parts of the brain into the affected area, thereby ameliorating potentially devastating effects (3). Although the high rate of variation in CoW morphology is well-known, the cause of the variation remains unclear. Both genetic factors and acquired hemodynamic modification are thought to play a role in the variation. In addition, variations in the CoW are thought to be correlated with ischemic stroke risk, aneurysm, leukoaraiosis volume and perioperative neurologic complications of carotid endarterectomy (4-7).

\section{INVESTIGATIVE METHODS}

Dissection was the initial avenue for CoW research, as it allows the structure of the CoW to be directly observed after embalming. Dissection is highly accurate and sensitive in detecting morphological variation in the CoW, as even vessels as fine as $0.1 \mathrm{~mm}$ can be clearly observed (8). However, dissection does have several limitations. For example, embalming may vary the structure of the CoW, causing a difference between real-life and postmortem measurements. It is also impossible to investigate collateral blood flow in the CoW in vivo. Moreover, dissection is not suitable for using in research regarding the association of the CoW with cerebral diseases, because it is hard to differentiate healthy individuals from patients with cerebral diseases. This fact accounts for disagreements in the literature on the prevalence of CoW variants. Recently, advanced mini- and noninvasive imaging techniques have facilitated in vivo observations of the CoW, enabling CoW research in both larger and more specific populations. Although the use of autopsy is gradually being replaced by imaging techniques, autopsy is still advocated given its ability to discern micro vessels and sophisticated CoW variants.

Advanced imaging techniques include conventional digital subtraction angiography (DSA), ultrasonography, magnetic resonance angiography (MRA), and computed tomography angiography (CTA). These methods can reveal one or more features of $\mathrm{CoW}$ in vivo, e.g., structural details, artery hemodynamics, downstream territorial perfusion and tissue metabolism. However, each technique has its own limitations. Due to its exceptional spatial and temporal resolution, conventional angiography is considered the gold standard for evaluation of the CoW; but it is also invasive and thus, while preferred for use intraoperatively, it is less appropriate for preoperative use. Ultrasonography is popular for the preoperative evaluation of stenosis carotid artery diseases, providing both anatomical and hemodynamic information. When combined with common carotid artery 


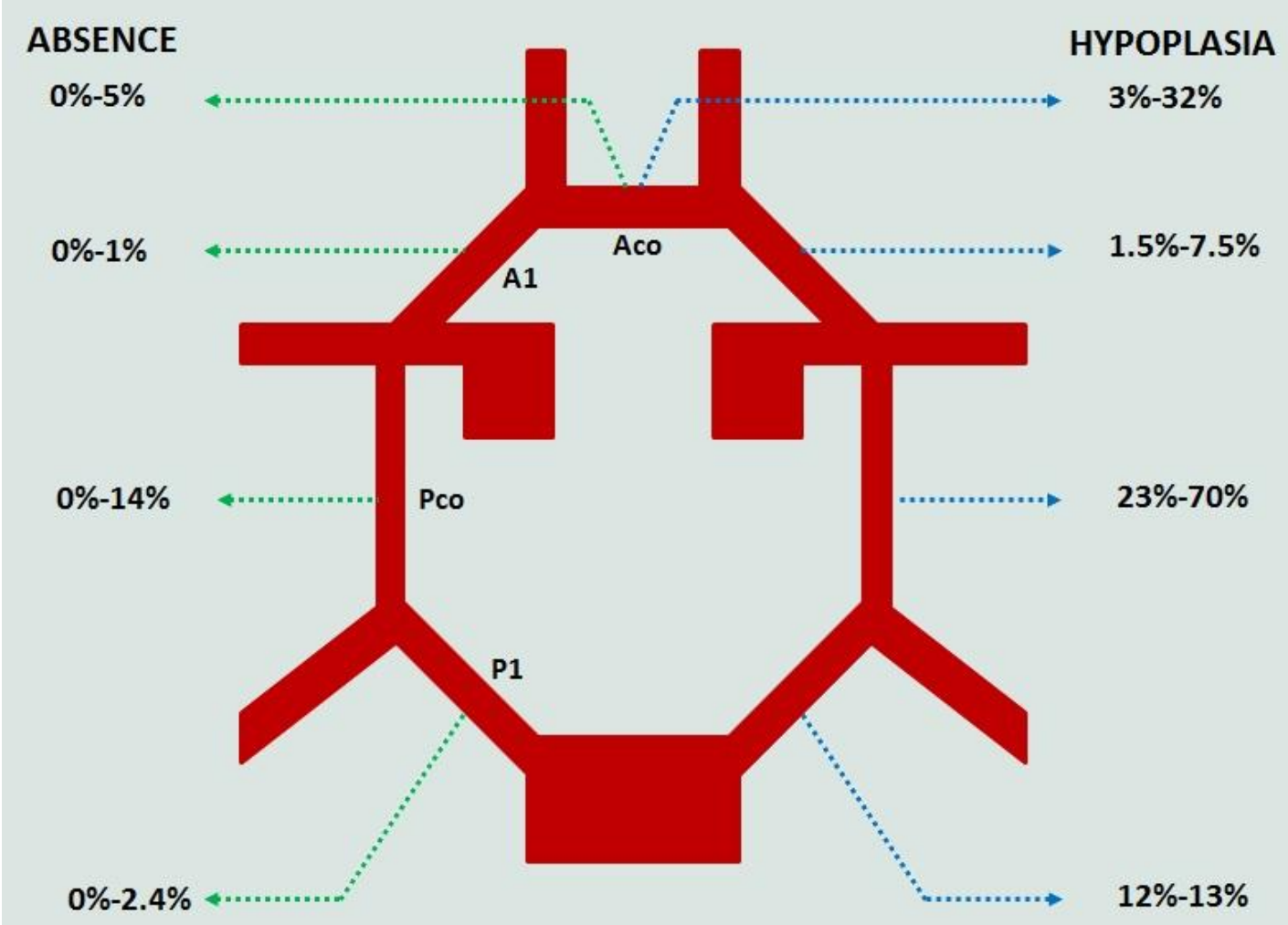

Figure 1. Schematic Drawing Labeled with the Prevalence of Absence and Hypoplasia of Each Segments of CoW from Dissection Literatures. (Modified from Varga A (12)).

compression, ultrasonography can effectively assess the collateral function of the CoW, but some segments, such as PCo are not visible (9). MR is the most common method used in current studies of the CoW, as it is both radiation-free and has high-resolution detection. Moreover i MR can provide novel insight regarding: the plaque characteristics of vessels, cerebral perfusion and tissue viability. Nevertheless, the 3-dimension time of flight MRA, the most popular MRA used in clinic, is affected by blood flow velocity of the vessel, so it is unsuitable for visualizing small vessels in the CoW with slow or turbulent flow and has a lower spatial resolution than that of DSA or CTA (2). As the least invasive examination with a maximum spatial resolution of 0.14 $\mathrm{mm}$, CTA can image the extra- and intracranial arteries in a single scan. In some cases, CTA has taken the place of DSA in the preoperative evaluation of these arteries, since it has high sensitivity and specificity for detecting stenosis of intracranial arterial disease, allowing stroke to be evaluated (10-12). Nonetheless, the conduction of
CTA raises concerns regarding ionizing radiation and it has been reported that each cerebral CTA incurs an $\sim 0.0026 \%$ increase in lifetime cancer risk (13). Lastly, many computer simulation models have been developed to calculate functional parameters of CoW, including one-, two-, or three-dimensional models, and patientspecific models (14).

\section{VARIATIONS OF THE COW}

The ability of the CoW to redirect blood flow is dependent on its integral circular form and the diameters of the component segments. In the data collected to date, morphometric variation in CoW is widely detected, however, the frequency of variants ranges between $10 \%$ and $85 \%$. This broad range may reflect different definitions of CoW variants, different methods used to measure variation, and differences in study populations (2). Using the development and status of each segment, i.e., 


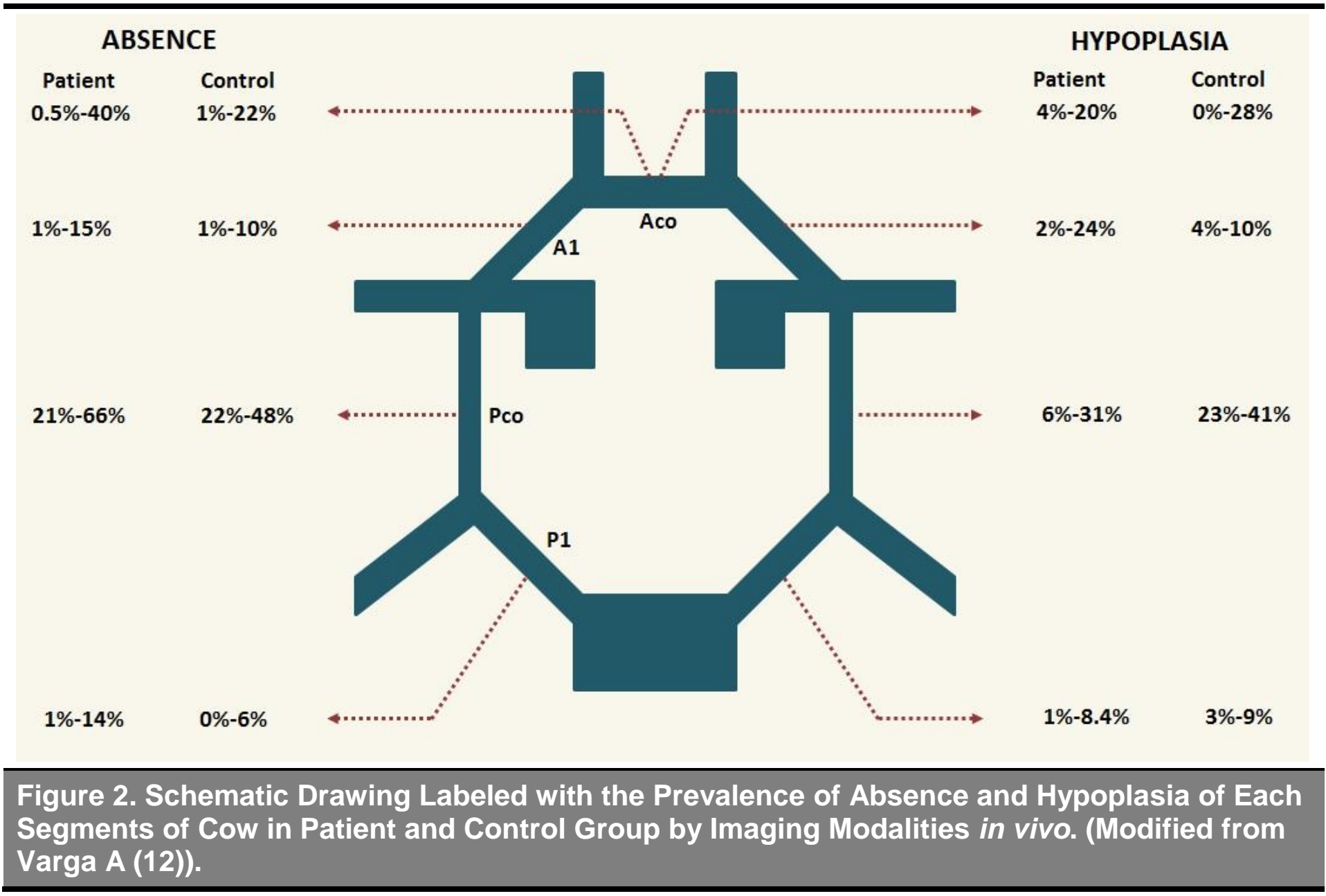

normal, hypoplastic or absent, CoW can be classified into more than 20 types. Nevertheless, knowledge of CoW segment development and variation is limited to the extent that no single reliable standard for classification exists.

The absence of any segment breaks the integrity of the CoW. As noted above, methodology may affect measures of variation frequency, as detection capability of methods for small vessels differs. For example, in dissection, absence is rarely found; the rate of absence in Pco is $0 \%-14 \%$ and the absence of Aco is $0 \%-5 \%$ based on dissection studies (Figure 1). In comparison, other methods indicate rates of absent Pco from 21\%-66\% and absent Aco from 0.5\%-40\% (12) (Figure 2).

The definition of "hypoplasia" is arbitrary. Typically, the threshold distinguishing normal from hypoplastic is set at $1 \mathrm{~mm}$ (where vessels $<1 \mathrm{~mm}$ in diameter are classified as hypoplastic), as some authors believe that $1 \mathrm{~mm}$ of diameter is inadequate to supply collateral flow, but some use a diameter of $0.3-0.6 \mathrm{~mm}$ as the criterion for hypoplasia. Exceptionally, $0.1 \mathrm{~mm}$ is used for the fetal brain. In ultrasonography studies, functional patency of the segments is defined by hemodynamic "tuning" during a common carotid artery C compression test (9). Studies suggest hypoplasia of Pco is most common; this variant has a prevalence of $23 \%-70 \%$ in dissections and $6 \%-41 \%$ using other methods $(4,15,16)$.

Fetal-type posterior cerebral artery (FTP) indicates those cases in which the diameter of the Pco is greater than or equal to the diameter of the ipsilateral P1. This variant also has a high prevalence; previous research estimates percentages ranging from $10 \%-40 \%$.

Populational factors and differences in studied cohort also contribute to unexplained differences in the prevalence of CoW variants. First, patient cohorts often demonstrate higher percentages of variants than healthy 
controls $(11,12)$. Second, the age of the study cohort may affect variant frequency. The majority of researchers suggest variations or anomalies demonstrated in the fetal period continue into postnatal life and age-related segment changes only arise if lesions occur; however, Van Overbeeke reported variations of P1 and Pco were significantly different between newborns and adults (1, $6,17)$. Third, although some studies have attempted to evaluate the distribution of $\mathrm{CoW}$ variations in different populations, racial differences in the CoW are unclear. De Silva reported ethnic differences in CoW variation among Caucasian, African and Asian populations (16). Moreover, compared to Westerners or Japanese, a higher prevalence of an incomplete posterior portion of the CoW is found in the Chinese population (18). Yet, in other reports, racial differences were not found $(4,5)$. In the majority of studies to date, no significant gender differences in the prevalence of anatomic variations have been observed $(2,18-21)$.

In general, variation of the CoW is accepted as occurring frequently, with the following factors rarely considered controversial: (i) the the prevalence of variation in the posterior circle is higher than in that of the anterior circle; (ii) variations in the CoW are correlated with cerebral or carotid vessel diseases.

\section{REASONS FOR VARIATION}

Many hypotheses have been suggested for why variations occur in the CoW; among these, two factors are thought to play important roles: genetic background and hemodynamics. The latter is related to the development or degeneration of embryo arteries, the functional significance of vascular segments, neck movement, and pathological lesions; thus, hemodynamics is a complex factor. The delicate hemodynamic 'tuning' of all component segments in the CoW act as the foundation for dynamic CoW variation. In early studies, Fields et al. suggested variations of the CoW are likely genetically determined, developed prenatally and persist in postnatal life (22). Milenkovid et al. found that fetal brains as early as 4 months had demonstrable differences between Aco and A1 segments, as well as Pco and P1 segments, and interpreted these as the embryonic form of the variation seen in adults (1). Vasovic et al. also noted variations in fetal characteristics and thus attributed variation to genetic differences (6). Although these authors emphasized the genic contribution to variants, research did not neglect the possibility of postnatal hemodynamic modifications. In much of the literature, postnatal biochemical or physical factors related to cerebral or carotid hemodynamic modification are deemed responsible for variations.

The development or degeneration of embryo arteries is also related to CoW variations. Puchades-Orts et al. found that in fetal brains, Pco maintains its maximum diameter until the primitive trigeminal artery disappears. If the trigeminal artery remains, the Pco becomes reduced in diameter, and may even disappear (23). Padget et al. suggested that persistence of the embryonic lateral branch of the posterior cerebral arteries causes FTP, leading to the use of 'fetal' in the name (19).

The correlation between the functional significance of segments and CoW variation has also been investigated. Van Overbeeke concluded that CoW variations were the result of increased functional demands for the rapid development of the occipital lobes (17). Subsequent studies have shown that the ACo may be the functionally most important collateral pathway (24), in keeping with the anterior portion of the CoW appearing to have a higher rate of integrity than the posterior portion. The Aco is helpful in retaining blood flow in the cerebral hemispheres and thus its absence or hypoplasia will be more costly in the event of cerebrovascular events than variation in the Pco.

In previous reports, Lazorthes et al. suggested that variation in CoW segments is rare in newborns up to one year because their greater amplitude of neck movement results in a more evenly distributed compression of all segments. In adults, movement of the neck and hemodynamic compression in CoW reduces with age, and atrophy of the segments may occur due to loss of function (6).

In general, it remains unclear whether genetic or acquired factors are responsible for $\mathrm{CoW}$ variation. We suggest that $\mathrm{CoW}$ variations are most likely the results of dynamic development of the segments; in Vasovic's words, "a theoretical model in prenatal and postnatal developmental phases." The association of variants and pathologic lesions are detailed in the next section.

\section{VARIATIONS AND PATHOLOGY LESIONS}

Studies of variation in the CoW focus mostly on its relationship with cerebral and carotid diseases, such as cerebral and carotid artery stenosis, ischemia stroke, aneu- 
rism and white matter lesions. This has been possible since the development of in vivo imaging techniques.

Computer simulations demonstrate that blood flow of the middle cerebral artery decreases with ipsilateral internal carotid artery (ICA) stenosis; in the absence of contralateral A1, when unilateral ICA stenosis occurs, the blood supply to the anterior part of the brain and to the whole brain hemisphere decreased to the minimum. Variations in the CoW can weaken collateral capability, and it is thus considered as one of the risk factors of ischemia stroke (14). In dissections, a higher prevalence of variation in the CoW was reported in stroke patients compared to controls. Following these early findings, imaging studies have provided new insights for the role of CoW variants in diseases. In studies on the completeness of CoW, Brunereau et al. reported that the CoWs were entirely complete in only $11 \%$ of 109 patients diagnosed with diverse neurologic disorders (25). Likewise, Waaijer et al. found that a compromised anterior circle segment, commonly coexisting with a compromised posterior segment, was detected more frequently in patients with symptomatic carotid artery stenosis than in controls (26). Schomer and Miralles showed that the absence and hypoplasia ( $<1 \mathrm{~mm}$ in diameter) of ipsilateral Pco and a non-functional Aco is a risk factor for ischemic cerebral infarction in patients with ICA occlusion $(27,28)$. Kluytman et al. recommended the pattern of collateral blood supply be used to evaluate cerebral hemodynamic status in patients with ipsilateral ICA occlusion; no collateral flow via CoW or flow via only the posterior communicating artery predicts deteriorated cerebral perfusion (29). Hoksbergen et al. assessed the collateral function of the CoW in an atherosclerotic population without cerebrovascular symptoms and found failure of this collateral pathway was caused by a hypofunctional anterior circle in $5 \%$ of subjects and a hypofunctional posterior circle in $45 \%$ subjects, including $4 \%$ in Aco and 38\% in Pco (9). Most recently, Varga et al. retrospectively analyzed CT and CTA data from 544 patients with severe stenosis of the ICA (> 70\% stenosis) and measured the percentage of hypoplasia, or absence, of individual segments; in all, except P1, this was higher in patients than in normal controls. Moreover, ICA stenosis was the only independent predictor of CoW configuration and severely compromised CoW were strongly correlated with brain ischemia (12). In contrast, Hartkamp et al. assessed the CoW configuration in 75 patients with ICA stenosis or occlusions and 100 normal subjects and found a higher percentage of complete CoW in patients (55\%) than in controls (36\%) (30). Arjal proposed that the existence of Pco may "steal" blood from the anterior circulation, resulting in ischemic stroke (31).

Leukoaraiosis is the neuro-radiological term for cerebral small-vessel disease. Hemodynamic modification is the main cause of leukoaraiosis and a partial reduction in leukoaraiosis is observed after artery revascularization operations. Ryan investigated the relationship between leukoaraiosis and completeness of CoW and found those with an incomplete CoW exhibited 58\% more white matter disease than those with a complete CoW. Similarly, frontal and occipital white matter lesions were correlated with an intact arterial and posterior circle, respectively (5). Saba assessed the association between CoW variations and the leukoaraiosis in different cerebral territories and concluded that the amount of leukoaraiosis was greater in subjects with more CoW variation that had severe internal carotid artery stenosis (7).

Researchers have also investigated whether the variations of $\mathrm{CoW}$ can predict intra- or post-operative neurologic complications of carotid endarterectomy. Banga demonstrated that the absence of both Aco and ipsilateral Pco carried a greater than 10-fold higher risk of transient ischemic attack and stroke occurring immediately after carotid endarterectomy with crossclamping without shunt protection (32). Likewise, Montisci found patients with two or more segments absent in the CoW have the highest risk (prevalence 60\%) of intolerance to cross-clamping during carotid endarterectomy (33).

In our opinion, an incomplete $\mathrm{CoW}$ increases the effects of low cerebral blood supply in patients with severe stenosis or occlusion ICA and increases the risk of ischemia in white matter disease. The anterior circle is recognized as the most important collateral pathway in the CoW. Moreover, the configuration of CoW can predict complications of carotid endarterectomy.

\section{FUTURE WORK}

Using artificial intelligence (AI) techniques, CTA and MRA data can be combined with deep learning algorithms to develop ways to segment the CoW and extract afferent and efferent vessels of CoW automatically and accurately. With these data, a 3D simulation mesh of vessels could be constructed, allowing CoW variants 
and stenosis of vessels to be evaluated automatically. Moreover, AI techniques can be used for automatic lesion identification. These imaging findings by AI can be used as an input feature in machines for predicting stroke prognosis. Recently, the predictive performance based on CT and MR data demonstrate superior to those conventional prognostication tools. In future, along with clinical data, the imaging information from AI technology is potential to more accurately predict ischemia stroke, provide risk stratification for stroke treatment and foresee the degree of post-stroke recovery.

\section{ARTICLE INFORMATION}

Author Affiliations: Research Institute for Translation Medicine on Molecular Function and Artificial Intelligence Imaging, and Department of Radiology, The First People's Hospital of Foshan, Affiliated Foshan Hospital of Sun Yat-sen University, Foshan 528000 , Guangdong, China (Wang W, Yang, Qiu, \& Pan); \& Center for Human Anatomy, School of Biomedical Sciences, University of Edinburgh, Edinburgh EH8 9AG, UK (Wang Q).

Author Contributions: Pan has full access to all of the data in the study and takes responsibility for the integrity of the data and the accuracy of the data analysis.

Study concept and design: Wang W, Pan.
Acquisition, analysis, or interpretation of data: Wang W, Wang Q, Yang, Qiu, \& Pan.

Drafting of the manuscript: Wang W. Critical revision of the manuscript for important intellectual content: BASE.

Statistical analysis: N/A.

Obtained funding: N/A.

Administrative, technical, or material support: Pan.

Study supervision: Pan.

Conflict of Interest Disclosures: Authors declared no competing interests of this manuscript submitted for publication.

Funding/Support: The study was supported by Science and Technology Innovation Project of Foshan (FSOAA-KJ218-1301-0021) to Dr. Pan.

Role of the Funder/Sponsor: The funder did not play any role in conducting and publishing the data.

How to Cite This Paper: Wang W, Wang Q, Yang G, Qiu M, Pan A. A review of the circle of Willis: Investigative methods, anatomical variations and correlated ischemic brain diseases. Sci Insigt. 2020; 33(2):159-166.

Digital Object Identifier (DOI): http://dx.doi.org/ 10.15354/si.20.re069.

Article Submission Information: Received, February 24, 2020; Revised: March 05, 2020; Accepted: March 20, 2020.

\section{REFERENCES}

1. Milenkovic Z, Vucetic R, Puzic M. Asymmetry and anomalies of the circle of Willis in fetal brain. Microsurgical study and functional remarks. Surg Neurol 1985; 24(5):563-570.

2. Krabbe-Hartkamp MJ, van der Grond J, de Leeuw FE, de Groot JC, Algra A, Hillen B, Breteler MM, Mali WP. Circle of Willis: Morphologic variation on three-dimensional time-of-flight MR angiograms. Radiology 1998; 207(1):103-111.

3. Manninen $\mathrm{H}$, Makinen $\mathrm{K}$, Vanninen $\mathrm{R}$, Ronkainen A, Tulla H. How often does an incomplete circle of Willis predispose to cerebral ischemia during closure of carotid artery? Postmortem and clinical imaging studies. Acta Neurochirurg 2009; 151(9):10991105.

4. Eftekhar B, Dadmehr M, Ansari S, Ghodsi M, Nazparvar B, Ketabchi E. Are the distributions of variations of circle of Willis different in different populations? - Results of an anatomical study and review of literature. BMC Neurol 2006; 6:22.

5. Ryan DJ, Byrne S, Dunne R, Harmon M, Harbison J. White matter disease and an incomplete circle of Willis. Int J Stroke 2015; 10(4):547-552.

6. Vasovic L, Milenkovic Z, Pavlovic S. Comparative morphological variations and abnormalities of circles of Willis: A minireview including two personal cases. Neurosurg Rev 2002; 25(4):247-251.

7. Saba L, Raz E, Fatterpekar G, Montisci R, di Martino M, Bassareo PP, Piga M. Correlation between leukoaraiosis volume and circle of Willis variants. J Neuroimag 2015; 25(2):226-231.

8. Kapoor K, Kak VK, Singh B. Morphology and comparative anatomy of circulus arteriosus cerebri in mammals. Anat Histol Embryol 2003; 32(6):347-355.

9. Hoksbergen AW, Legemate DA, Ubbink DT, Jacobs MJ. Collateral variations in circle of willis in atherosclerotic population assessed by means of transcranial color-coded duplex ultrasonography. Stroke 2000; 31(7):1656-1660.

10. Hata A, Yanagawa M, Honda O, Kikuchi N, Miyata T, Tsukagoshi S, Uranishi A, Tomiyama N. Effect of
Matrix Size on the Image Quality of Ultra-high-resolution CT of the Lung: Comparison of $512 \times 512,1024 \times$ 1024, and $2048 \times 2048$. Acad Radiol 2018; 25(7):869-876.

11. Nguyen-Huynh MN, Wintermark $M$, English J, Lam J, Vittinghoff E, Smith WS, Johnston SC. How accurate is CT angiography in evaluating intracranial atherosclerotic disease? Stroke 2008; 39(4):1184-1188.

12. Varga A, Di Leo G, Banga PV, Csobay-Novak C, Kolossvary M, Maurovich-Horvat $\mathrm{P}$, Huttl $\mathrm{K}$. Multidetector CT angiography of the Circle of Willis: Association of its variants with carotid artery disease and brain ischemia. Eur Radiol 2019; 29(1):46-56.

13. Malhotra A, Wu X, Chugh A, Mustafa A, Matouk CC, Gandhi D, Sanelli P. Risk of radiation-induced cancer from computed tomography angiography use in imaging surveillance for unruptured cerebral aneurysms. Stroke 2018:STROKEAHA118022454.

14. Zhu G, Yuan Q, Yang J, Yeo JH. The role of the circle of Willis in internal carotid artery stenosis and anatomical 
variations: A computational study based on a patient-specific threedimensional model. Biomed Engineer Online 2015; 14:107.

15. Klimek-Piotrowska W, Rybicka M, Wojnarska A, Wojtowicz A, Koziej M, Holda MK. A multitude of variations in the configuration of the circle of Willis: An autopsy study. Anat Sci Int 2016; 91(4):325-333.

16. De Silva KR, Silva R, Amaratunga $D$, Gunasekera WS, Jayesekera RW. Types of the cerebral arterial circle (circle of Willis) in a Sri Lankan population. BMC Neurol 2011; 11:5.

17. Van Overbeeke JJ, Hillen B, Tulleken CA. A comparative study of the circle of Willis in fetal and adult life. The configuration of the posterior bifurcation of the posterior communicating artery. J Anat 1991; 176:45-54.

18. Li Q, Li J, Lv F, Li K, Luo T and Xie P. A multidetector CT angiography study of variations in the circle of Willis in a Chinese population. J Clin Neurosci 2011; 18(3):379-383.

19. Kapoor K, Singh B, Dewan LI. Variations in the configuration of the circle of Willis. Anat Sci Int 2008; 83(2):96106.

20. Macchi C, Catini C, Federico C, Gulisano M, Pacini P, Cecchi F, Corcos L, Brizzi E. Magnetic resonance angiographic evaluation of circulus arteriosus cerebri (circle of Willis): A morphologic study in 100 human healthy subjects. Ital J Anat Embryol 1996; 101(2):115-123.

21. Horikoshi T, Akiyama I, Yamagata Z, Sugita M, Nukui H. Magnetic reso- nance angiographic evidence of sexlinked variations in the circle of Willis and the occurrence of cerebral aneurysms. J Neurosurg 2002; 96(4):697703.

22. Fields WS. Aortocranial occlusive vascular disease (stroke). Clin Symposia $1974 ; 26(4): 3-31$.

23. Puchades-Orts A, Nombela-Gomez M, Ortuno-Pacheco G. Variation in form of circle of Willis: Some anatomical and embryological considerations. Anat Rec 1976; 185(1):119-123.

24. Chrzanowska G, Mierzwa J. Variation of the cerebral arterial circle (of Willis) in man. Folia Morphol 1979; 38(3):365-373.

25. Brunereau L, Levy C, Arrive L, Bousson V, Marsot-Dupuch K, Bousser MG, Tubiana JM. Anatomy of the circle of Willis with 3D time of flight magnetic resonance angiography and analysis of partitions. J Radiol 1995; 76(9):573-577.

26. Waaijer A, van Leeuwen MS, van der Worp HB, Verhagen HJ, Mali WP, Velthuis BK. Anatomic variations in the circle of Willis in patients with symptomatic carotid artery stenosis assessed with multidetector row CT angiography. Cerebrovas Dis 2007; 23(4):267-274.

27. Schomer DF, Marks MP, Steinberg GK, Johnstone IM, Boothroyd DB, Ross MR, Pelc NJ, Enzmann DR. The anatomy of the posterior communicating artery as a risk factor for ischemic cerebral infarction. $N$ Eng $\mathrm{J}$ Med 1994; 330(22):1565-1570.

28. Miralles M, Dolz JL, Cotillas J,
Aldoma J, Santiso MA, Gimenez A, Capdevila A, Cairols MA. The role of the circle of Willis in carotid occlusion: assessment with phase contrast MR angiography and transcranial duplex. Eur J Vas Endovas Surg 1995; $10(4): 424-430$.

29. Kluytmans M, van der Grond J, van Everdingen KJ, Klijn CJ, Kappelle LJ, Viergever MA. Cerebral hemodynamics in relation to patterns of collateral flow. Stroke 1999; 30(7):1432-1439.

30. Hartkamp MJ, van Der Grond J, van Everdingen KJ, Hillen B, Mali WP. Circle of Willis collateral flow investigated by magnetic resonance angiography. Stroke 1999; 30(12):26712678.

31. Arjal RK, Zhu T, Zhou Y. The study of fetal-type posterior cerebral circulation on multislice CT angiography and its influence on cerebral ischemic strokes. Clin Imag 2014; 38(3):221225.

32. Banga PV, Varga A, Csobay-Novak C, Kolossvary M, Szanto E, Oderich GS, Entz L, Sotonyi P. Incomplete circle of Willis is associated with a higher incidence of neurologic events during carotid eversion endarterectomy without shunting. J Vas Surg 2018; 68(6):1764-1771.

33. Montisci R, Sanfilippo R, Bura R, Branca C, Piga M, Saba L. Status of the circle of Willis and intolerance to carotid cross-clamping during carotid endarterectomy. Eur J Vas Endovas Surg 2013; 45(2):107-112. 